\title{
Live attenuated Salmonella typhimurium vaccines delivering SaEsxA and SaEsxB via type III secretion system confer protection against Staphylococcus aureus infection
}

Chen Xu ${ }^{1}$, Bao-zhong Zhang ${ }^{1}$, Qiubin Lin ${ }^{1}$, Jian Deng ${ }^{1}$, Bin Yu' ${ }^{1}$ Smriti Arya ${ }^{1}$, Kwok-Yung Yuen ${ }^{2}$ and Jian-Dong Huang ${ }^{1,3,4^{*}}$ (D)

\begin{abstract}
Background: Staphylococcus aureus (S. aureus) causes a wide range of infectious diseases in human and animals. The emergence of antibiotic-resistant strains demands novel strategies for prophylactic vaccine development. In this study, live attenuated S. enterica subsp. enterica serotype Typhimurium (S. Typhimurium) vaccine against S. aureus infection was developed, in which Salmonella Pathogenesis Island-1 Type 3 Secretion System (SPI-1 T3SS) was employed to deliver SaEsxA and SaEsxB, two of ESAT-6-like (Early Secreted Antigenic Target-6) virulence factors of S. aureus.

Methods: Antigens SaEsXA and SaEsXB were fused with the N-terminal secretion and translocation domain of SPI-1 effector SipA. And cytosolic delivery of Staphylococcal antigens into macrophages was examined by western blot. BALB/c mice were orally immunized with $S$. Typhimurium-SaEsXA and S. Typhimurium-SaEsxB vaccines. Antigen-specific humoral and Th1/Th17 immune responses were examined by ELISA and ELISPOT assays 7-9 days after the 2nd booster. For ELISPOT assays, the statistical significance was determined by Student's t test. The vaccine efficacy was evaluated by lethal challenge with two S. aureus clinical isolates Newman strain and USA 300 strain. Statistical significance was determined by Log rank (Mantel-Cox) analysis. And a $P$ value of $<0.05$ was considered statistically significant.

Results: Oral administration of S. Typhimurium-SaEsxA and S. Typhimurium-SaEsxB vaccines induced antigen-specific humoral and Th1/Th17 immune responses, which increased the survival rate for vaccinated mice when challenged with S. aureus strains.

Conclusions: The newly developed S. Typhimurium-based vaccines delivering SaEsXA and SaEsxB by SPI-1 T3SS could confer protection against $S$. aureus infection. This study provides evidence that translocation of foreign antigens via Salmonella SPI-1 T3SS into the cytosol of antigen presenting cells (APCS) could induce potent immune responses against pathogens.
\end{abstract}

Keywords: Live attenuated S. Typhimurium vaccine, T3SS, MRSA, S. aureus, SaEsxA, SaEsxB

* Correspondence: jdhuang@hku.hk

${ }^{1}$ School of Biomedical Sciences, The University of Hong Kong, Hong Kong,

SAR, China

${ }^{3}$ HKU-Shenzhen Institute of Research and Innovation, Shenzhen, China

Full list of author information is available at the end of the article

(c) The Author(s). 2018 Open Access This article is distributed under the terms of the Creative Commons Attribution 4.0 International License (http://creativecommons.org/licenses/by/4.0/), which permits unrestricted use, distribution, and reproduction in any medium, provided you give appropriate credit to the original author(s) and the source, provide a link to the Creative Commons license, and indicate if changes were made. The Creative Commons Public Domain Dedication waiver (http://creativecommons.org/publicdomain/zero/1.0/) applies to the data made available in this article, unless otherwise stated. 


\section{Background}

Staphylococcus aureus, a commensal of human skin and nares, is also a human pathogen with dual intracellular and extracellular lifestyle $[1,2]$. S. aureus infection causes a broad range of diseases, from skin infections to life-threatening diseases, including pneumonia, endocarditis, and sepsis et al. [1, 3]. The recent emergence of multidrug-resistant $S$. aureus strains, such as methicillinresistant $S$. aureus (MRSA), exerts a huge clinical burden and makes the infections much more difficult to treat [4-6]. However, a prophylactic vaccine against $S$. aureus is not available. Many active and passive immunization vaccines, such as StaphVAX, V710, Pagibaximab, tefibazumab, Veronate, Aurograb and AltaStaph, ended in failure in clinical trials [7-13], which might be attributed to several reasons. Firstly, cell-surface antigens were targeted by all the seven vaccines, and the elicited antibodies against surface components might promote bacterial aggregation, causing possible tissue damage, ischaemia, multi-organ failure and death [7, 14]. Secondly, failures of the five passive immunization strategies might be ascribed to an overemphasis on humoral immunity, rather than the cooperation of humoral and cellular immunity [7, 15].

Secreted virulent factors are also targeted for vaccine development and exhibit effective protection against $S$. aureus infection diseases [16-18]. Among them, SaEsxA and SaEsxB are two ESAT-6-like virulence factors required for persistence and spread of $S$. aureus in the infected host [19]. SaEsxA and SaEsxB were first identified in the culture filtrates of Mycobacteria tuberculosis ( $M$. tuberculosis) and found to be able to stimulate T-cell immune responses $[20,21]$. In 2005, SaEsxA and SaEsxB were experimentally verified to be secreted from $S$. aureus and involved in abscess formation. S. aureus mutant lacking EsxA or EsxB showed defects during $S$. aureus infection in murine model [19]. SaEsxA and SaEsxB also regulate the apoptosis and release of intracellular $S$. aureus from the infected epithelial cells [2].

During recent years, protein subunit vaccines targeting SaEsxA and SaEsxB were shown to prevent invasive S. aureus infection through induction of Th1- and Th17-biased immunity [22]. Administration of a four-component vaccine 4C-Staph/alum including SaEsxA/B chimera was demonstrated to protect mice from $S$. aureus infection in four murine models, including kidney abscess, peritonitis, skin and pneumonia models [23, 24]. In addition, a novel adjuvant T7-alum targeting TLR7 was formulated with the four component vaccine 4C-Staph (4C-Staph/T7-alum), which showed outperformance over the previous 4C-Staph/alum [25]. The immunologic protection was attributed to the cooperation of vaccine-specific antibodies, CD4+ T cells and IL-17 [25]. This study emphasizes on the cooperation of humoral and cellular immunity for protection against $S$. aureus infection.
Live attenuated $S$. Typhimurium strains have been employed to deliver recombinant foreign antigens with various approaches [26]. Among these, SPI-1 T3SS has been engineered for the cytosolic delivery of foreign antigens to induce efficient immune responses against cancer and infectious diseases $[27,28]$. SPI-1 T3SS-mediated cytosolic delivery of antigens could significantly enhance the accessibility of antigens to MHC class-I antigen presenting pathway, and effectively activate CD8+ T cell-mediated immunity, which is important to remove intracellular pathogens such as S. aureus [27, 29-31]. In addition, utilization of Salmonella T3SS for protein expression can prevent formation of inclusive bodies and cellular degradation of target proteins, which happened during protein expression by other gram-negative expression systems [32].

SPI-1 T3SS is a needle-like apparatus to directly translocate effectors into mammalian host cells, which facilitates bacteria invasion and pathogenesis [33-35]. This process is mediated by different chaperones targeting the N-terminal tags of the cognate effectors [36]. In this study, N-terminal domain of SipA (1-169aa) was fused with staphylococcal antigens to achieve the translocation. SipA is a bi-functional SPI-1 T3SS effector. On one hand, SipA functions extracellularly to mediate trans-epithelial migration of polymorphonuclear neutrophils [37], via engaging certain host surface receptors to activate protein kinase $\mathrm{C}(\mathrm{PKC})$ and subsequently induce the apical release of pathogen-elicited epithelial chemoattractant (PEEC) [37-39]. On the other hand, SipA could also be translocated into the cytosol of host cells by T3SS, inducing actin cytoskeleton rearrangement and membrane ruffle, which are required for bacterial uptake $[40,41]$.

In this study, SPI-1 T3SS was utilized to deliver Staphylococcal antigens SaEsxA and SaEsxB into the cytosol of host cells. Our results showed that oral immunization with these strains could elicit multifaceted immune responses in mice, which conferred protection against $S$. aureus. To the best of our knowledge, this is the first development of live attenuated $S$. Typhimurium based vaccine against $S$. aureus infection. Besides, this study also provides information that N-terminal signal peptide of SipA (1169aa) could be utilized as molecular carrier for the cytosolic delivery of foreign proteins via SPI-1 T3SS, with the support of the cognate chaperon invB.

\section{Methods}

\section{Bacterial strains and growth conditions}

Bacteria strains and plasmids used in this study were listed in Table 1.

Salmonella enterica serovar Typhimurium aroA-deleted ( $\triangle$ aroA) strain SL7207 was kindly provided by Dr. B.A.D Stocker [42], which was utilised as the parent for genomic knockouts. ML21 is an isogenic double knockout ( $\triangle$ aroA, $\Delta$ pryF), derived from SL7207. ML88 is a triple knockout 
Table 1 Bacterial strains and plasmids used in this study

\begin{tabular}{|c|c|c|}
\hline Strains & Description & Reference \\
\hline SL7207 & $\Delta$ aroA & Lab stock \\
\hline ML21 & $\Delta$ aroA $\Delta$ pyrF & Lab stock \\
\hline ML86 & ML21 $\Delta$ sipB & Lab stock \\
\hline ML88 & ML21 $\triangle$ invA & Lab stock \\
\hline N19 & ML21 carrying PagC-invB-sipA-SaEsxA (ColE1 ori) & This study \\
\hline N20 & ML21 carrying PagC-invB-sipA-SaEsxB (ColE1 ori) & This study \\
\hline N106 & ML21 carrying empty plamsid (ColE1 ori) & This study \\
\hline N80 & $\begin{array}{l}\text { ML86 ( } \Delta \text { sipB) carrying PagC-invB-sipA-SaEsxA } \\
\text { (ColE1 ori) }\end{array}$ & This study \\
\hline N158 & $\begin{array}{l}\text { ML88 ( } \Delta \text { invA) carrying PagC-invB-sipA-SaEsxA } \\
\text { (ColE1 ori) }\end{array}$ & This study \\
\hline N160 & $\begin{array}{l}\text { ML86 ( } \Delta \text { sipB) carrying PagC-invB-sipA-SaEsxB } \\
\text { (ColE1 ori) }\end{array}$ & This study \\
\hline N161 & $\begin{array}{l}\text { ML88 ( } \Delta \text { invA) carrying PagC-invB-sipA-SaEsxB } \\
\text { (ColE1 ori) }\end{array}$ & This study \\
\hline
\end{tabular}

( $\Delta$ aroA, $\Delta$ pryF, $\Delta$ invA), derived from ML21. The isogenic knockouts were constructed using $\lambda$ Red-recombineering method [43].

$S$. Typhimurium and E. coli strains were cultured in Luria-Bertani (LB) broth or LB agar supplemented with appropriate antibiotics. For secretion assay, S. Typhimurium was cultured in SPI-1 Inducing LB (0.3 M $\mathrm{NaCl}$ ) [36]. S. aureus strains were grown in Brain Heart Infusion Broth (BHI, Sigma-Aldrich) and BHI agar.

Antibiotics were used at the final concentrations: streptomycin $(50 \mu \mathrm{g} / \mathrm{mL})$, chloramphenicol $(25 \mu \mathrm{g} / \mathrm{mL})$, ampicillin $(100 \mu \mathrm{g} / \mathrm{mL})$ and kanamycin $(50 \mu \mathrm{g} / \mathrm{mL})$.

\section{Mammalian cell culture}

Murine RAW264.7 macrophages were purchased from ATCC and cultured in RPMI 1640 medium (Gibco) with $10 \%$ Fetal Bovine Serum (FBS, Gibco). Cell cultures were maintained at $37{ }^{\circ} \mathrm{C}$ in a humidified atmosphere containing $5 \% \mathrm{CO}_{2}$.

\section{Construction of plasmids}

Plasmid pCASP (-sicP-sptP) (Genbank: \#EF179157), which is based on pPROTet.133 backbone $\left(\mathrm{Cm}^{\mathrm{R}}\right.$, ColE1) (BD Clonetech), was generously provided by Prof. Christopher Voigt [36]. Plasmids with alternative secretion tag/chaperone pairs, including pCASP-invB-sipA, pCASP-invB-sopE2, pCASP-invB-sopA, and pCASP-sigEsopB were constructed by overlap extension PCR, as previously described [36]. Briefly, secretion tags, chaperones and PsicA were amplified from $S$. Typhimurium genomic DNA with the primers listed in Table 2. The pcr products were fused by overlap extension PCR, followed by double enzyme digestion (HindIII and XhoI) and ligation into the plasmid pCASP to replace PsicA-sicP-sptP. For the simplicity of further genetic modification, PacI restriction enzyme site was introduced between PsicA and chaperones.

SaEsxA and SaEsxB were PCR amplified from the genomic DNA of S.aureus ATCC 25923 with primers including $6 \times$ his tag. After double enzyme digestion (BamHI and NotI), DNA fragments SaEsxA-his and SaEsxB-his were ligated into the BamHI and NotI restriction sites of pCASPs in frame with different signal peptides.

The pagC promoter (PpagC) was PCR amplified from the genomic of $S$. Typhimurium genomic DNA. After digested with restriction enzyme XhoI and PagC, PpagC was inserted into plasmids PsicA-invB-sipA-SaEsxA and PsicA-invB-sipA-SaEsxB to replace PsicA.

\section{Secretion assays}

Secretion assay was performed according to the method described previously with modifications [36]. Briefly, bacteria strains were streaked on LB agar plates supplemented with appropriate antibiotics and cultured at $37{ }^{\circ} \mathrm{C}$ overnight. Single colonies were inoculated in $4 \mathrm{~mL}$ LuriaBertani (LB) broth media, supplemented with chloramphenicol and streptomycin and grown overnight at $220 \mathrm{rpm}$. The next morning, bacteria cultures were diluted to an OD600 of 0.01 in $4 \mathrm{~mL}$ fresh LB broth, supplemented with chloramphenicol and streptomycin and grown for $2 \mathrm{~h}$ at $220 \mathrm{rpm}$. Then the bacteria cultures were diluted at the ratio of $1: 10$, into $4 \mathrm{~mL}$ inducing $\mathrm{LB}$ $(0.3 \mathrm{M} \mathrm{NaCl})$ supplemented with chloramphenicol and streptomycin, and were grown for $8 \mathrm{~h}$ at $160 \mathrm{rpm}$. Media and bacteria were separated by centrifugation at $4700 \mathrm{~g}$ for $10 \mathrm{~min}$. Supernatants were filtered with 0 . $22 \mu \mathrm{m}$ syringe filters (Sartorius). The supernatant samples were concentrated 20X by trichloroacetic acid (TCA) precipitation. Briefly, $100 \%(w / v)$ TCA was added to the supernatants to a final concentration of $20 \%$, and incubated at $4{ }^{\circ} \mathrm{C}$ overnight. The mixture was centrifuged at $17,000 \mathrm{~g}$ for $5 \mathrm{~min}$ at $4{ }^{\circ} \mathrm{C}$. And the pellet was washed with $500 \mu \mathrm{L}$ cold acetone. Pellets were dried at room temperature for about $5 \mathrm{~min}$ and loaded onto sodium dodecyl sulfate polyacrylamide gel electrophoresis (SDS-PAGE) after re-suspended in $1 \times$ SDS-PAGE loading buffer. On the other hand, the bacteria pellets were washed with cold PBS once and re-suspended in PBS containing proteinase inhibitors, which is a mixture of Aprotinin from bovine lung (Sigma), Leupeptin hydrochloride (Sigma) and Pepstatin A (Sigma). The bacterial cells were sonicated for $2 \mathrm{~min}$ in $4 \mathrm{~s}$ pulses with $35 \%$ amplitude, followed by centrifugation at $19,000 \mathrm{~g}$ for $20 \mathrm{~min}$. The supernatants were collected, and mixed with $6 \times$ SDS-PAGE loading buffer for SDS-PAGE.

\section{Bacterial infection of RAW264.7 macrophages and translocation assays}

The detection of translocated heterologous antigens was carried out as described previously with modifications 


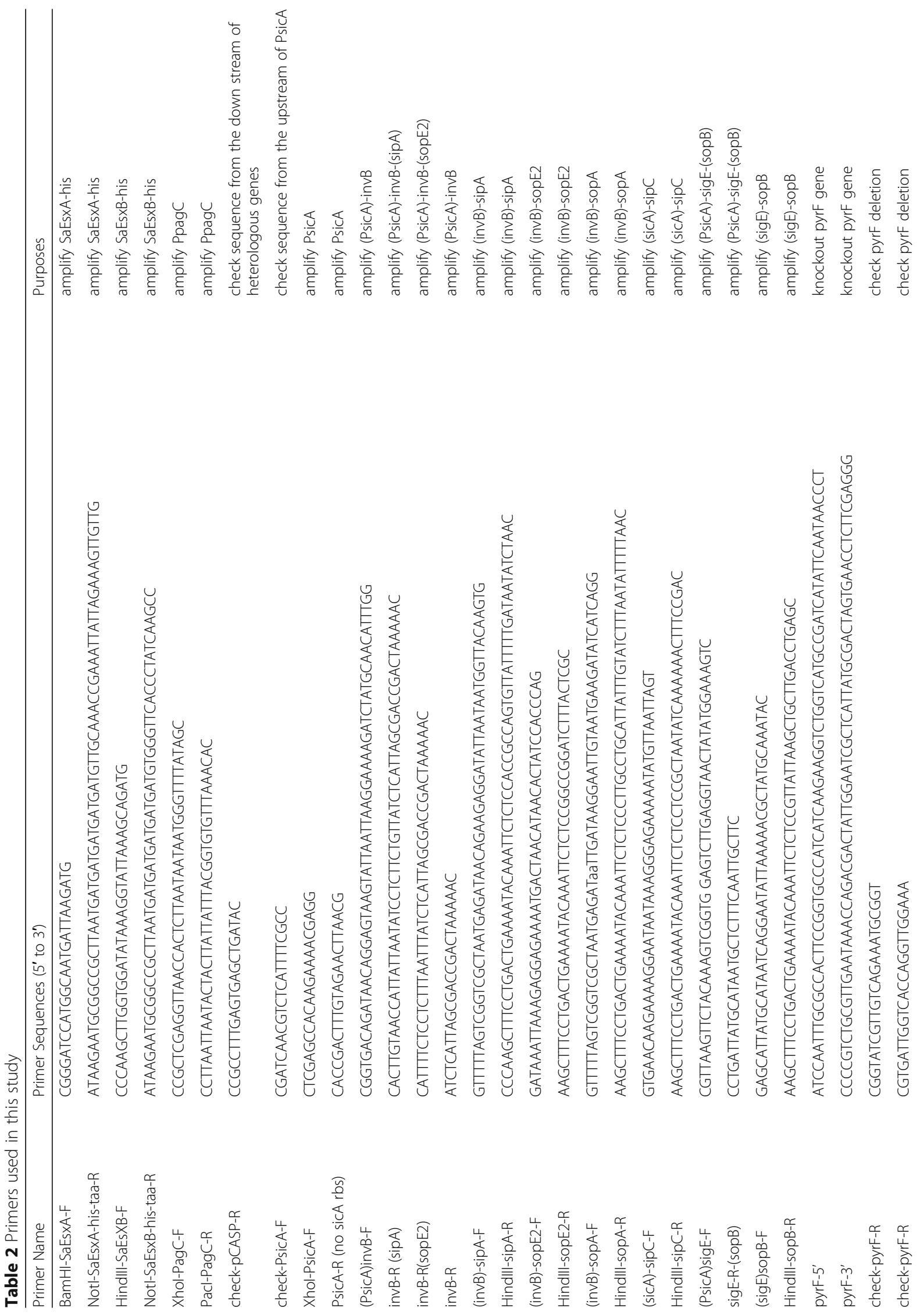




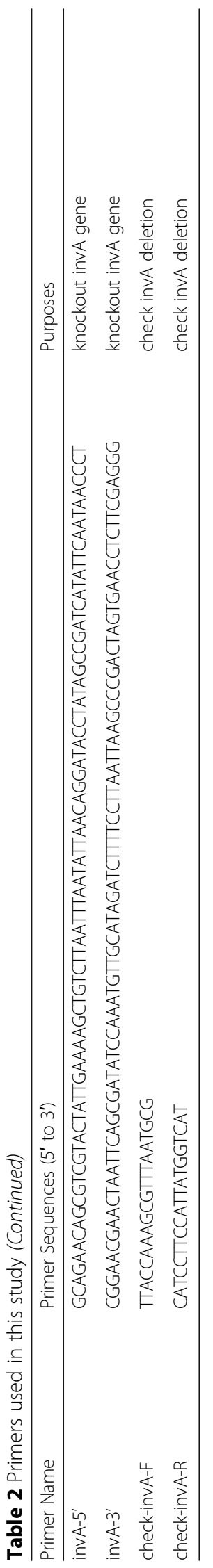


[44]. Briefly, bacteria were cultured overnight at $37^{\circ} \mathrm{C}$ in LB broth supplemented with appropriate antibiotics, and subcultured at 1:30 dilution for $2 \mathrm{~h}$ until OD600 reached 1.5. Then bacteria were harvested by centrifugation at $17,000 \mathrm{~g}$ for $2 \mathrm{~min}$, washed once with sterile PBS, and re-suspended in RPMI 1640 medium with 10\% FBS. When Raw264.7 macrophages reached to 70-80\% confluency in $60 \mathrm{~mm}$ tissue culture dishes, bacteria were added at a MOI of 100 and infected the cells at $37{ }^{\circ} \mathrm{C}$ for $3 \mathrm{~h}$ in $3 \mathrm{~mL}$ RPMI 1640 medium with 10\% FBS. After infection, cells were washed thrice with PBS, and cultured in RPMI 1640 medium with 10\% FBS containing $100 \mu \mathrm{g} / \mathrm{mL}$ gentamicin for another hour. After washed with cold PBS once, cells were lysed by lysis buffer which is PBS containing $0.1 \%$ Triton X-100 and proteinase inhibitors, and detached with a rubber cell scraper. Cell soluble fraction (containing bacteria-free cytosol) and insoluble fraction (containing insoluble cell components and intracellular bacteria) were separated by centrifugation at $19,000 \mathrm{~g}$ for $20 \mathrm{~min}$. The soluble fraction samples were further filtered by $0.22 \mu \mathrm{m}$ filter (Sartorius) to get rid of bacteria, and then concentrated by TCA precipitation, as described above. Both soluble and insoluble fractions were used for western blot analysis.

\section{SDS-PAGE and western blot}

SDS-PAGE and western blot was conducted following standard methods. Protein samples were mixed with SDS-loading dye, boiled for $10 \mathrm{~min}$ and analyzed by $12 \%$ SDS-PAGE. Separated proteins were transferred to PVDF membrane and blocked by $5 \% \mathrm{w} / v$ skim milk/TBST (Tris Buffered Saline containing 0.1\% Tween 20) for $1 \mathrm{~h}$ at room temperature. Membranes were incubated with mouse anti$6 \times$ His tag monoclonal antibody (HIS.H8, MA1-21315) at 1:2000 v/v dilution at $4{ }^{\circ} \mathrm{C}$ overnight. After washed with TBST, membranes were incubated with Horseradish peroxidase (HRP)-conjugated sheep anti-mouse IgG (GE Healthcare Life Sciences, NA931-1ML) at room temperature for $1 \mathrm{~h}$. After washed with TBST, the membranes were developed with Immobilon Western Chemiluminescent HRP Substrate (MERCK Millipore) and the signal was analyzed by Bio-Rad ChemiDocTM MP Imaging system.

\section{Animal immunization and lethal challenge experiments}

All animal experiments were approved by the Committee on the Use of Live Animals in Teaching and Research at the Laboratory Animal Unit of the University of Hong Kong (CULATR 3569-15).

5- to 6- week-old male BALB/c mice were purchased from the Laboratory Animal Unit of the University of Hong Kong, and were kept under specific-pathogen-free conditions. The immunization procedure was similar to that described previously [26]. Briefly, BALB/c mice received three doses of $\mathrm{S}$. Typhimurium vaccine by oral gavage on Day 1, Day 8 and Day 22. $4 \mathrm{~h}$ after mice were deprived of food and water, $100 \mu \mathrm{L} 3 \%$ NaHCO3 was provided orally to neutralize the gastric acid. Then each mouse was vaccinated by $5+$ E10 colony form unit (CFU) of freshly cultured and PBS-washed bacterial cells. The vaccination was conducted with a feeding needle with round bottom.

Ten days after the secondary booster, immunized mice were challenged through intravenously injecting $5 \mathrm{E}+07 \mathrm{CFU}$ of $S$. aureus USA 300 strain (communityassociated MRSA [CA-MRSA]) or 5E + 07 CFU of Newman strains (methicillin-susceptible $S$. aureus [MSSA]). Mortality and clinical signs of mice were monitored for 14 days.

Animal experiments were repeated at least twice with similar results.

\section{Enzyme-linked immunosorbent assay (ELISA)}

Seven days after the second booster, $\sim 100 \mu \mathrm{L}$ blood was collected from the tail vein using Microvette ${ }^{\bullet}$ CB 300 LH (Sarstedt), and stored at room temperature for $1 \mathrm{~h}$, followed by centrifugation at $2000 \mathrm{~g}$ for $10 \mathrm{~min}$ at room temperature. Sera were collected for ELISA and could be stored at $-80{ }^{\circ} \mathrm{C}$ until use. Meanwhile, $\sim 70 \mathrm{mg}$ of feces were collected and suspended in $400 \mu \mathrm{L}$ PBS. Then the mixture was vortexed and centrifuged at $10000 \mathrm{~g}$ for $15 \mathrm{~min}$ and the supernatants were collected for ELISA or stored at $-80{ }^{\circ} \mathrm{C}$.

ELISA was performed as previously described [22]. Briefly, ELISA plates (Nuc, Roskilde, Denmark) were coated with recombinant SaEsxA (rSaEsxA) or rSaEsxB at the concentration of $1 \mu \mathrm{g} / \mathrm{mL}$ in coating buffer (carbonate-bicarbonate, $\mathrm{pH}=9.6$ ) overnight at $4{ }^{\circ} \mathrm{C}$. Then ELISA plates were blocked with $5 \% \mathrm{w} / v$ skim milk/TBST at $37{ }^{\circ} \mathrm{C}$ for $2 \mathrm{~h}$. Mouse sera were serially diluted with $5 \% \mathrm{w} / \mathrm{v}$ skim milk/TBST at threefold and were added into the wells. While fecal extracts were serially diluted at twofold. After incubating for $1 \mathrm{~h}$ at $37^{\circ} \mathrm{C}$, the plates were washed five times with TBST. HRP-rabbit antimouse IgG2a secondary antibody (Invitrogen, 610,220), HRP-rabbit anti-mouse IgG1 secondary antibody (Thermo Scientific, PA1-86329) and HRP-goat anti-mouse IgA secondary antibody (Invitrogen, 62-6720) were used according to the manufacturer's instructions. After incubating for $1 \mathrm{~h}$ at $37{ }^{\circ} \mathrm{C}$, the plates were washed six times with TBST. For color development, plates were incubated with tetramethylbenzidine (TMB) substrate solution for $15 \mathrm{~min}$ at room temperature, followed by adding stop solution (2 $\mathrm{M} \mathrm{H}_{2} \mathrm{SO} 4$ ). Absorbance at $450 \mathrm{~nm}$ was recorded by ELISA plate reader. The antibody titre was expressed as the inverse of the greatest dilution of sera which showing over twofold OD450 readout than that of the control sample at the same dilution. The assays were conducted in duplicate and the experiment were repeated twice. 


\section{Enzyme-linked immunospot (ELISPOT) assay}

Eight or nine days after the second booster, mice (3-4 mice/group) were euthanized with Pentobarbitone sodium (100-150 mg/kg, i.p.) and spleens were harvested, homogenised and forced through $70 \mu \mathrm{m}$ cell strainer (BD) with the ends of sterile syringe plungers [45]. The splenocytes were obtained by centrifugation at $250 \mathrm{~g}$. Erythrocytes were lysed with ACK buffer for 5 min at room temperature. After washing once with PBS, splenocytes were resuspended in RPMI 1640 medium with 10\% FBS, and filtered with $70 \mu \mathrm{m}$ cell strainer (BD) to get rid of cell aggregates.

Interferon-gamma (IFN- $\gamma$ ) and Interleukine-17A (IL17A) ELISPOT assays were conducted with Mouse IFN- $\gamma$ ELISPOT kit (R\&D Systems) and Mouse IL-17 ELISpot Kit (R\&D Systems) respectively, according to the manufacturer's instructions. Briefly, splenocytes, stimulated by antigen rSaEsxA or rSaEsxB at the concentration of $2 \mu \mathrm{g} /$ $\mathrm{mL}$, were plated at the concentration of $5 \mathrm{E}+05$ cells/well in duplicate for $20 \mathrm{~h}$ at $37{ }^{\circ} \mathrm{C}$. Then plates were washed and incubated with biotinylated anti-IFN- $\gamma$ or anti-IL-17A antibody overnight at $4{ }^{\circ} \mathrm{C}$. After washing the plates, streptavidin-Alkaline phosphatase was added and incubated for $2 \mathrm{~h}$ at room temperature. At last, the plates were incubated with substrate BCIP/NBT Chromogen for 0.5-1 $\mathrm{h}$ at room temperature for color development. The spots were counted using an immunospot reader system.

\section{Statistical analysis}

Data were presented as the means \pm SEM. For ELISPOT assays, the statistical significance was determined by Student's $t$ test. For survival rates in the lethal challenge experiments, statistical significance was determined by Log rank (Mantel-Cox) analysis. GraphPad Prism 6 was used to conduct these analyses. And a $P$ value of $<0.05$ was considered statistically significant.

\section{Results}

\section{SPI-1 T3SS-dependent secretion of SaEsxA and SaEsxB}

Attenuated $S$. Typhimurium strains were engineered to express and secret antigens SaEsxA and SaEsxB via SPI-1 T3SS. As shown in Fig. 1a, SaEsxA and SaEsxB were fused with five different $\mathrm{N}$-terminal secretion peptides of SPI-1 effectors under PsicA promoter, which is the endogenous promoter of SPI-1 effector sipA. Basal transcription level of PsicA is quite low, however upon induction with high osmolarity and low aeration, SPI-1 T3SS is established and activated [46], with a 200-fold increase of transcription efficiency [36]. His-tag at the C-terminal of antigens was used for detection purpose. Additionally, previous articles indicated the importance of specific chaperones of the secretion tags for secretion and translocation efficiency $[29,36]$, thus cognate chaperones was co-expressed with fusion antigens under PsicA.
To investigate the secretion efficiency of heterologous antigens, secretion assays were conducted, in which attenuated $S$. Typhimurium strain ML21 carrying a series of plasmids was cultured in low salt $\mathrm{LB}$, and shifted to high salt $\mathrm{LB}$ media $(0.3 \mathrm{M} \mathrm{NaCl})$ to induce the secretion via SPI-1 T3SS. Expression and secretion were examined in vitro by western blot. Fig. 1b showed that all the five fusion proteins could be detected in bacterial cell lysates. Secretion efficiency of SipA-SaEsxA was comparatively high, which could be detected without TCA precipitation. After TCA precipitation, other SaEsxA fusion proteins were also detected in the LB media. For SaEsxB, among all five SaEsxB fusion proteins with different signal peptides, only SipA-SaEsxB was detected to be expressed and secreted from $S$. Typhimurium in inducing LB after TCA precipitation (Fig. 1c). Thus, the most optimal chaperon/tag pair for SaEsxA and SaEsxB was invB/sipA, which was utilized for further vaccine development. The expression and secretion level of SipA-SaEsxA is much higher than that of SipASaEsxB (data not shown). In order to activate potent immune responses in vivo, the SPI-1 T3SS-inducible promoter PsicA was replaced with in vivo-inducible promoter of phoP-activated gene $\mathrm{C}$, designated as PpagC, which could lead to stable and increased expression of foreign antigens, and efficiently enhance immunogenicity in mice [47].

In order to demonstrate that the secretion of SaEsxA and SaEsxB is dependent of SPI-1 T3SS, rather than unspecific bacterial cell lysis, isogenic $S$. Typhimurium $\Delta$ invA mutant ML88 was constructed for secretion assay. Gene invA encodes a critical structural component of SPI-1 T3SS, thus $\Delta$ invA mutant is defective in SPI-1-mediated secretion [44]. As shown in Fig. 1d and Fig. 1e, secretion of SaEsxA and SaEsxB was eliminated in $\Delta$ invA strain, although fusion proteins could be detected in the bacterial cell lysates. The ghost band in Fig. 1d might be the target protein released from lysed bacterial cells, rather than actively secreted via T3SS. Taken together, these data indicated that the protein can be secreted in a SPI-1 T3SS-dependent way.

\section{Cytosolic delivery of SaEsxA and SaEsxB into macrophages}

To explore the potential of $S$. Typhimurium to translocate heterologous antigens into the cytosol of host cells, Raw264.7 macrophages were infected with $S$. Typhimurium strain ML21, $\triangle$ invA mutant ML88 and $\triangle$ sipB mutant ML86, carrying plasmids encoding SipA-SaEsxA and SipA-SaEsxB. Gene sipB encodes one of the three translocators of SPI-1 T3SS. SipB is indispensable for the translocation of the effectors into the cytosol of host cells [48], which is as same as gene invA. After infection at multiplicity of infection (MOI) of 100 for $3 \mathrm{~h}$, the infected macrophages were lysed with $0.1 \%$ Triton $\mathrm{X}-100$. Presence of foreign antigens in both Triton X-100- 

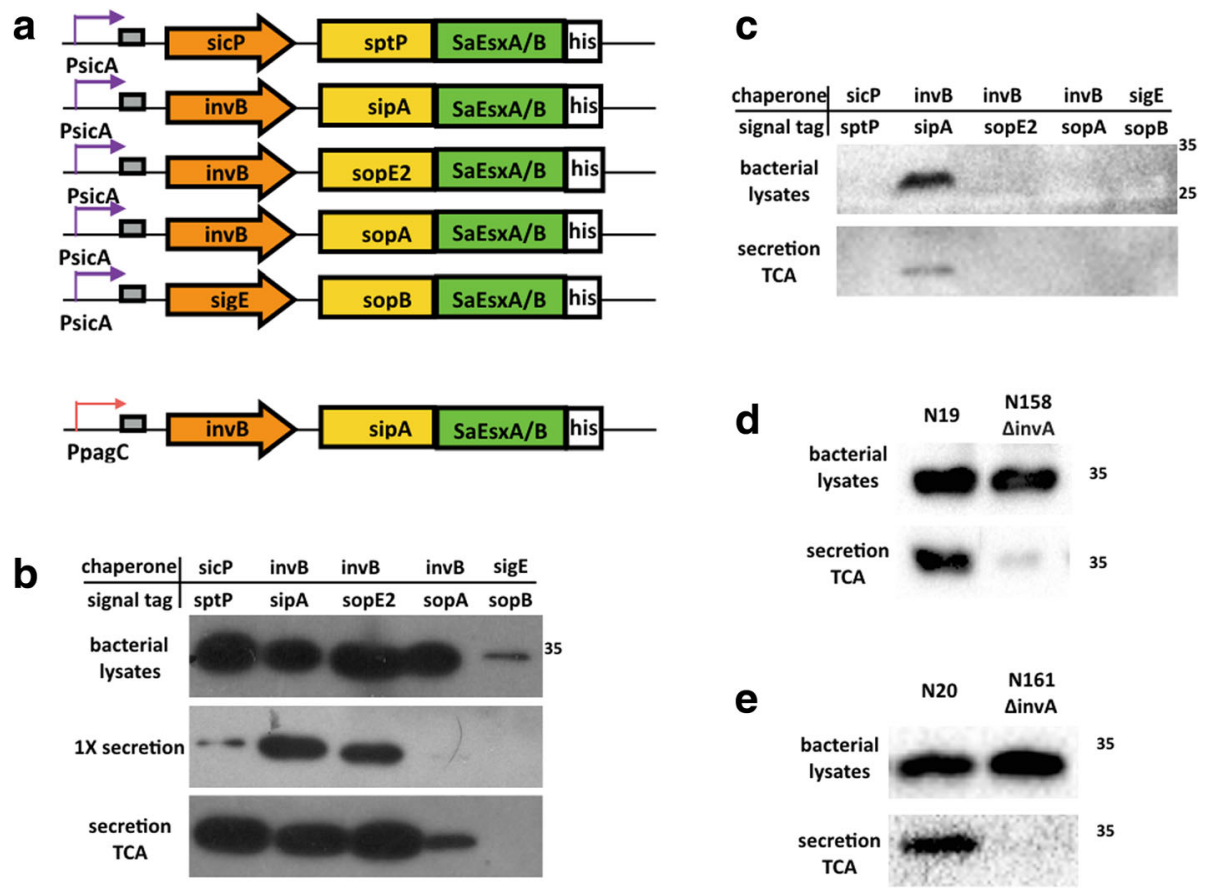

Fig. 1 SPI-1 T3SS-dependent secretion of SaEsxA and SaEsxB fusing with different secretion tags. SaEsxA and SaEsxB were fused with N-terminal secretion tags, and were expressed with cognate chaperones under PsicA promoter. $6 \times$ his tag at C-terminus was used for detection (a). Secretion assays were conducted for SaEsXA (b) and SaEsxB (c) to identify the optimal secretion tag/chaperone pairs. And the secretion of SipA-SaEsxA (d) and SipA-SaEsxB (e) from S. Typhimurium strain ML21 and isogenic $\Delta$ invA mutant was compared. For bacterial cell lysates, an equivalent of $200 \mu \mathrm{L}$ bacterial culture was used for SDS-PAGE. And for "1× secretion", an equivalent of $13 \mu \mathrm{L}$ culture was used and exposure time of western blot extended to 1 min. For "secretion TCA", secreted proteins were further 20-fold concentrated by TCA precipitation, and an equivalent of $400 \mu \mathrm{L}$ culture was used

insoluble fraction containing intracellular bacteria and Triton X-100-soluble fraction containing bacteria-free cytosolic proteins were examined by western blot. As shown in Fig. 2a, fusion protein SipA-SaEsxA was detected in the intracellular bacteria for $S$. Typhimurium strain N19, N158 ( $\triangle$ invA) and N80 $(\Delta$ sipB $)$, which are ML21, $\Delta$ invA mutant and $\Delta$ sipB mutant harboring plasmid encoding SipA-SaEsxA. Whereas, translocation of SipA-SaEsxA into the cytosol of macrophages was only detected from strain N19, but not from N158 ( $\triangle$ invA) and N80 $(\Delta \operatorname{sipB})$. Similarly, expression of SipA-SaEsxB can be detected in the intracellular bacteria for $S$. Typhimurium strain N20, N161 $(\Delta$ invA) and N160 $(\Delta$ sipB $)$, however translocation of SipA-SaEsxB was only detected in the bacteria-free cytosol of macrophages after infection with $S$. Typhimurium strain N20 (Fig. 2b). Thus, heterologous antigens SaEsxA and SaEsxB in fusion with Nterminal SipA (1-169aa) could be translocated into the

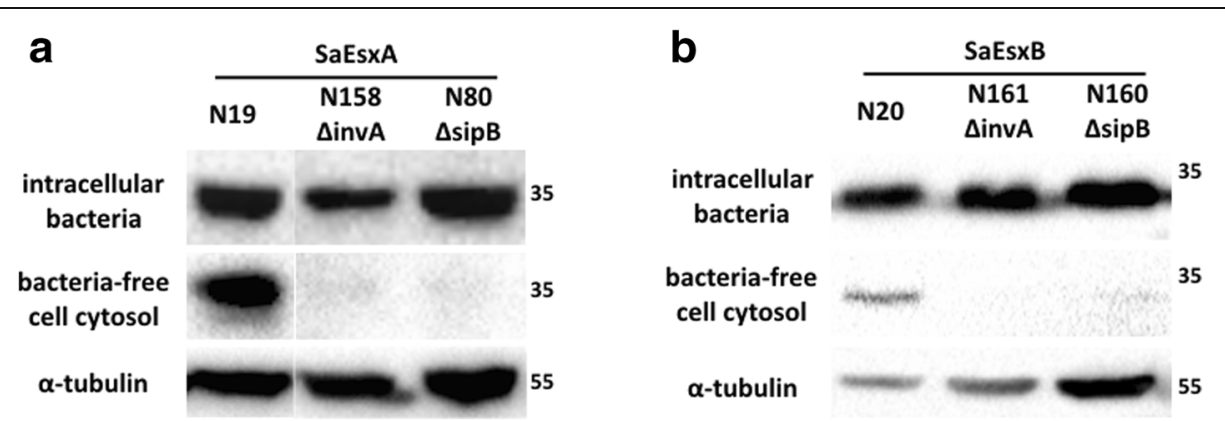

Fig. 2 Translocation of SaEsXA and SaEsxB fusion proteins into macrophages. Raw264.7 macrophages were infected with ML21, isogenic $\triangle$ invA mutant and $\Delta$ sipB mutant, carrying plasmids encoding SipA-SaEsXA (a) and SipA-SaEsXB (b) for 3 h. After lysis with 0.1\% Triton X-100, infected macrophages were separated into Triton X 100-insoluble fraction containing intracellular bacteria and Triton X 100-soluble fraction containing bacteria-free cell cytosol. a-tubulin was used as an internal control for protein loading 
cytosol of infected cells, which is dependent on SPI-1 T3SS. This data also indicates that $\mathrm{N}$-terminal secretion and translocation domain of SipA could serve as a molecular carrier for cytosolic delivery of heterologous antigens, mediated by chaperone invB.

\section{Stimulation of antigen-specific humoral immune responses}

To study the immunogenicity of $S$. Typhimurium-based vaccine in vivo, $B A L B / c$ mice were immunized with strain N19, N20 and the vector control strain N106, by oral gavage on Day 1, Day 8 and Day 22. Seven days after the 2nd booster, blood was collected and antigen-specific humoral immune responses in sera were examined by ELISA. As shown in Fig. 3a, mice immunized with N19 generated high level of rSaEsxA-specific IgG1 and IgG2a antibodies (average titers: 16,000), indicating that vaccination with N19 stimulated both Th1- and Th2-type responses. The rSaEsxB-specific IgG1 antibody titer of mice immunized with N20 was approximately 37,000, while rSaEsxB-specific IgG2a antibody titer was approximately 13,000. This result suggested that SaEsxB delivered by $S$. Typhimurium vaccine stimulated Th2-biased immune responses.

Considering the importance of secretory $\operatorname{IgA}(\mathrm{sIg} A)$ in immune exclusion and homeostasis maintenance of $S$. aureus in the intestine tract [49], sIgA antibodies against $\mathrm{rSaEsxA}$ and $\mathrm{rSaEsxB}$ in fecal extract of vaccinated mice were also determined by ELISA. The results indicated the presence of anti-rSaEsxA sIgA in the fecal extract of $4 / 5$ mice vaccinated with N19. While for the mice vaccinated with N20, anti-rSaEsxB sIgA was detected in only $1 / 10$ mice (Fig. $3 b$ ). No antigen-specific antibodies were detected from the mice immunized with the control strain N106.

Raw data are available in Additional file 1: Table S1.
Stimulation of antigen-specific IFN- $\gamma^{+}$and IL- $17 A^{+} \mathrm{T}$ cell immune responses

Previous research indicated that Th1 and Th17 cellmediated immunity contributes to the protection against $S$. aureus infection $[50,51]$, therefore we investigated whether SaEsxA and SaEsxB delivered by attenuated $S$. Typhimurium could elicit specific cellular immune responses. After the 2nd booster, IFN- $\gamma$ and IL-17A ELISPOT assays were conducted with splenocytes collected from vaccinated mice. The result in Fig. $4 \mathrm{a}$ and $4 \mathrm{~b}$ showed that after stimulated with rSaEsxA, splenocytes from mice vaccinated with $\mathrm{N} 19$ had more IFN- $\gamma$ - and IL-17A-secreting cells than that of mice treated with control strain N106 and PBS. Similarly, mice immunized with $\mathrm{N} 20$ showed strong rSaEsxB-specific IFN- $\gamma^{+}$and IL-17A ${ }^{+} \mathrm{T}$ cell responses (Fig. $4 \mathrm{c}$ and $4 \mathrm{~d}$ ). Taken together, these data suggest that vaccination by N19 and N20 strains could elicit antigen-specific Th1- and Th17-biased cellular immune responses.

Raw data are available in Additional file 2: Table S2.

\section{Protection against lethal challenge}

To evaluate the protection efficacy conferred by different vaccinations, ten days after the second booster, vaccinated mice were challenged with two clinical $S$. aureus strains at $5 \mathrm{E}+07 \mathrm{CFU}$ by intravenous injection through the tail vein. Mice were monitored for 14 days. The results in Fig. 5a revealed that oral administration of N20 significantly reduced the death of mice after lethal challenge with $S$. aureus USA 300 (44.4\% survival; $P=0.0105$, log rank Mantel-Cox test), in comparison the mice in N106 control group. Whereas, vaccination with N19 improved the survival rate to $22.2 \%(P=0.0881, \log$ rank Mantel-Cox test). In addition, when challenged with $S$. aureus Newman strain, even though all mice died within 10 days, vaccination with N19 and N20 could stimulate protective
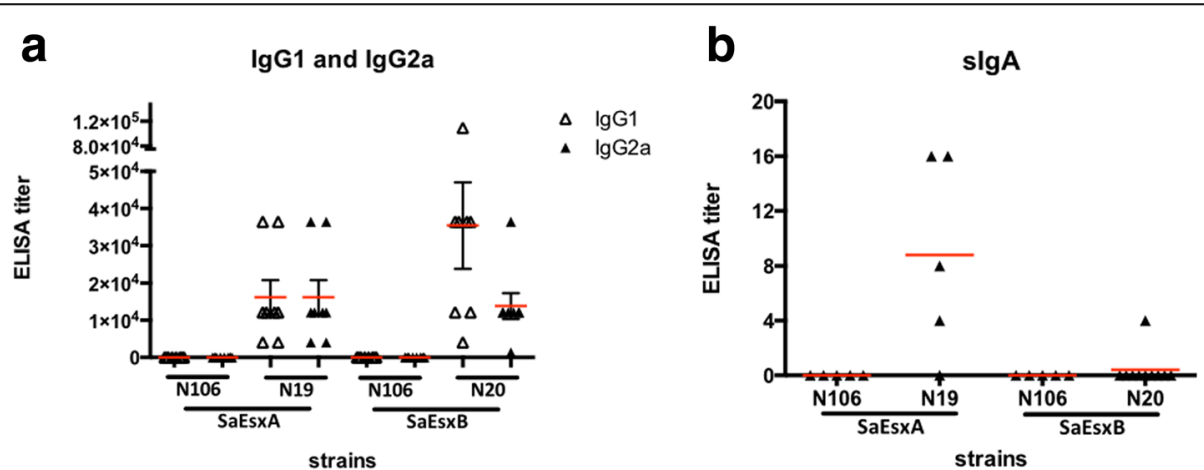

Fig. 3 Evaluation of humoral and mucosal immune responses against rSaEsXA and rSaEsxB by ELISA. Mice were immunized with N106, N19 and N20 strains ( $n=8$ /group). SaEsxA and SaEsxB were delivered via SPI-1 T3SS by N19 and N20, respectively. Seven days after the second booster, antigen-specific serum $\operatorname{lgG} 1$ and $\lg G 2 \mathrm{a}(\mathbf{a})$, as well as fecal slgA (b) were examined 
a

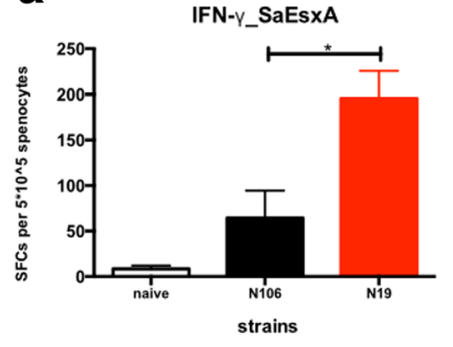

C

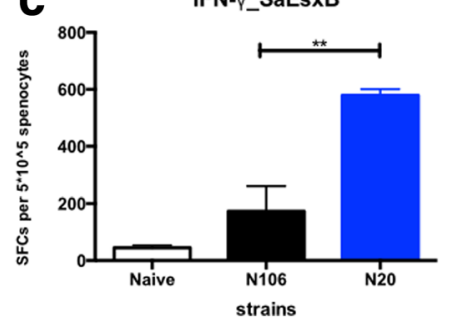

b
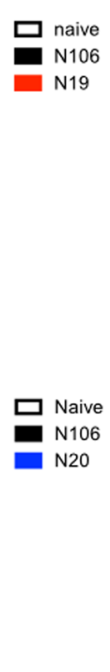

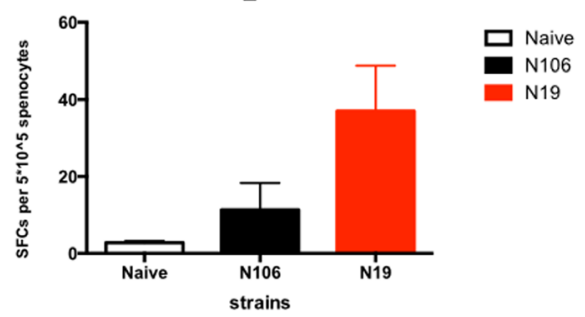

d

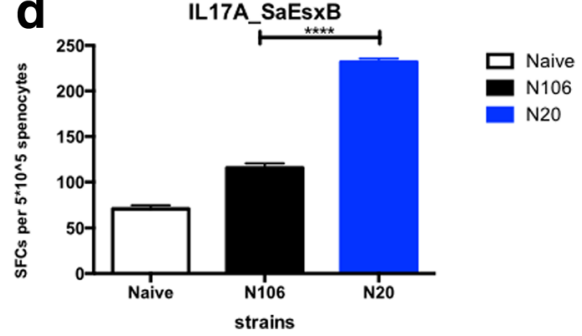

Fig. 4 Evaluation of cellular immune response against rSaEsxA and rSaEsxB by ELISPOT. Mice were immunized with PBS, N106, N19, and N20 strains ( $n=3-4$ /group). Eight or nine days after the second booster, splenocytes were prepared and incubated with specific antigens for $20 \mathrm{~h}$. IFN- - -producing cells with stimulator rSaEsXA (a) and rSaEsXB (c) were detected by IFN- $\gamma$ ELISPOT assay. And for IL-17A-producing cells stimulated with rSaEsxA (b) and rSaEsxB (d), IL-17A ELISPOT assays were conducted. SFC, Spot-Forming Cells. Results of one of two experiments are shown. For statistical analysis, student's t test was used. Data were represented as means \pm SEM
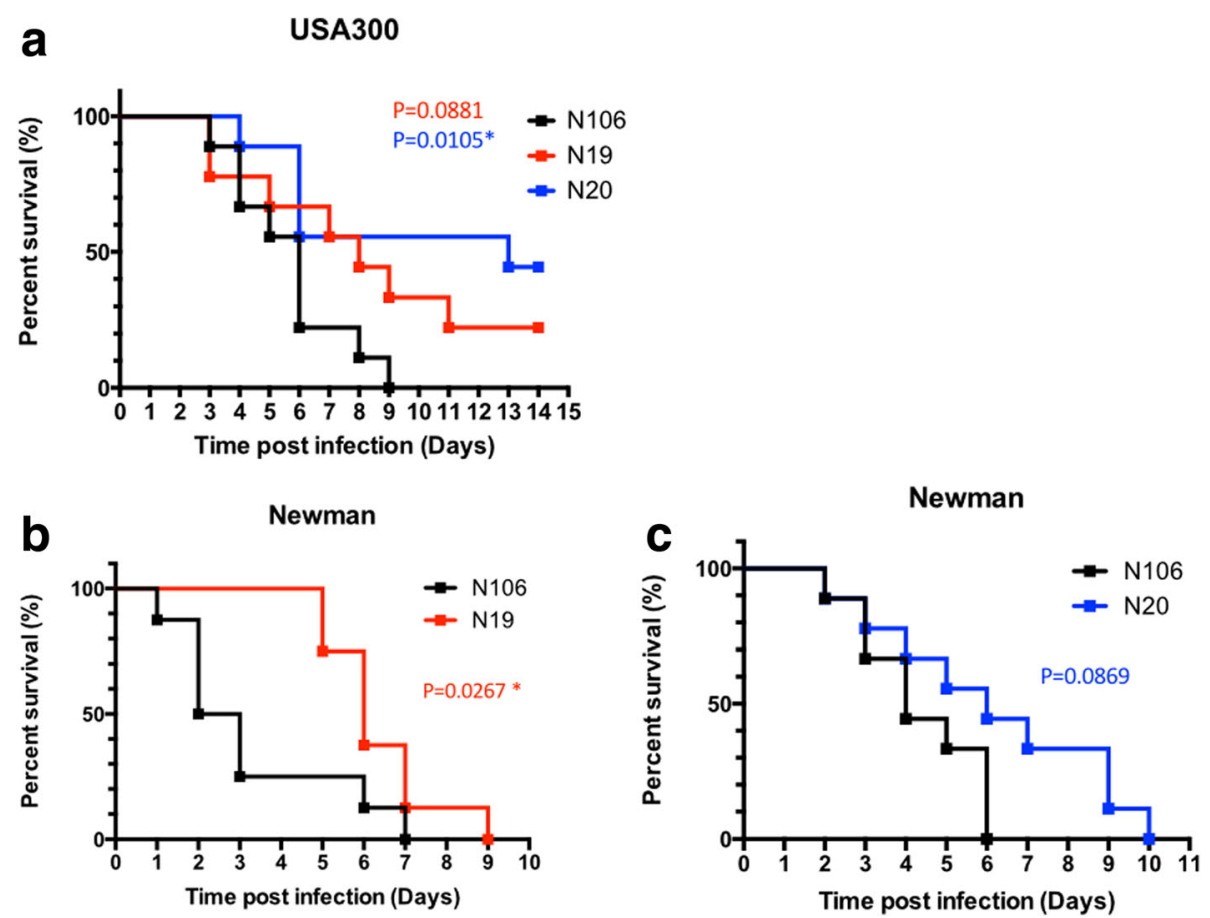

Fig. 5 Survival curves of vaccinated BALB/C mice after lethal challenge with two clinical S. aureus strains. Mice immunized with N106, N19 and N20 were challenged with S. aureus USA 300 (a) or Newman strains (b, c)(5E +07 CFU) by intravenous injection ( $n=8-9 /$ group). Mice were monitored for 14 days. Log rank (Mantel-Cox) tests were conducted to compare the protection induced by different vaccination. Results from one of two representative experiments are shown 
effect and postpone death, as shown in Fig. 5b and 5c. (for N19, $P=0.0267$; for N20 $P=0.0869$, log rank MantelCox test).

Raw data are available in Additional file 3: Table S3.

\section{Discussion}

In this study, live attenuated $S$. Typhimurium vaccine against $S$. aureus was developed, in which staphylococcal antigens SaEsxA and SaEsxB were delivered into the cytosol of host cells via SPI-1 T3SS. Oral administration of these vaccines elicited multi-faceted immune responses, including both humoral and cell-mediated immune responses, which confer protection against two clinical $S$. aureus strains.

Specific antibodies including SaEsxA- and SaEsxBspecific IgG in serum, as well as SaEsxA-specific sIgA in fecal extract were detected in vaccinated mice. During earlier stage of $S$. aureus infection, initial elimination of extracellular S. aureus was largely dependent on opsonophagocytosis facilitated by specific antibodies and complement components in serum [52]. Those SaEsxA- and SaEsxB-specific IgG antibodies might be crucial for binding and neutralizing $S$. aureus while it is in the extracellular stage. However, previous study on protein subunit vaccines demonstrated that antibodies generated against SaEsxA and SaEsxB were not effective against $S$. aureus infection, suggesting that cellular immune responses may be important to achieve good efficacy [22].

Antigen-specific stimulation of IFN- $\gamma$ secretion was detected in vaccinated mice, indicating the activation of Th1-type immune response. This data is consistent with previous reports that oral administration of $S$. Typhimurium vaccine elicited Th1-biased immune responses $[1,27,53,54]$, which effectively fight against intracellular pathogens [55, 56]. As accumulated evidence suggested, $S$. aureus is not exclusively extracellular bacterium, but is also capable of invading host cells. Upon invasion, the intracellular $S$. aureus can persist and replicate in the phagosomes, while some can escape from the phagosomes and enter the cytoplasm $[57,58]$. The intracellular fate of $S$. aureus in professional phagocytes was postulated to form a dissemination route of infection [59]. Cytotoxic $\mathrm{T}$ cells, natural killer cells and cytokine IFN- $\gamma$ are required to eliminate the intracellular bacteria $[60,61]$. Therefore, the stimulated IFN- $\gamma$ secretion could play important role in the clearance of intracellular $S$. aureus.

Th17 responses were also induced by $S$. Typhimurium delivering SaEsxA and SaEsxB, as suggested by the detection of IL-17A ${ }^{+}$splenocytes. Many studies indicated the importance of Th17 responses in the protection against a wide range of bacterial and fungal pathogens [62]. Th17 cells could mediate serotype-independent protection, through several mechanisms, such as recruiting neutrophils and macrophages to mucosal sites, activating $B$ cell antibody responses [63, 64]. Besides, long-life Th17 effector memory cells were detected in mucosal tissues, indicating their potential role against pathogens [65].

Previous researchers have shown that numerous viral and bacterial antigens were delivered into the cytosol of host cells via SPI-1 T3SS, presented to MHC class I-restricted antigen processing pathway, and subsequently activated CD8+ T cell-mediated immune responses [27, 29-31]. Our data showed the secretion and translocation of SipA-SaEsxA and SipA-SaEsxB fusion proteins into cytosol of Raw264.7 macrophages in vitro and stimulation of IFN- $\gamma$-dependent cellular immunity in vivo. Therefore, we hypothesized that the staphylococcal antigens delivered by $S$. Typhimurium carrying plasmids pCASP-invB-sipA-SaEsxA/B were presented by MHC class I molecules to CD8+ T cells, which could fight against $S$. aureus at intracellular stage.

Several limitations in the current study should be mentioned. Firstly, even though the two $S$. Typhimurium vaccines targeting either SaEsxA or SaEsxB could elicit significant antigen-specific immunity, neither of them conferred effective protection against the challenge with both S. aureus USA 300 and Newman strains. As suggested by previous articles that, in comparison with singleantigen vaccine, multivalent vaccines could evoke broad immune responses, and confer protection against different serotypes of $S$. aureus. Thus, to improve $S$. Typhimurium vaccine, a multivalent vaccine should be designed to facilitate the simultaneous delivery of SaEsxA, SaEsxB and other antigens. Secondly, antigen expression level in the recombinant attenuated Salmonella is one of the most fundamental determinants of vaccine efficacy. It's observed that the expression level of SaEsxB in $S$. Typhimurium is comparatively low, thus codon optimization of $\mathrm{SaEsxB}$ should be conducted to ensure optimal amount of SaEsxB expressed and delivered into host cells to achieve more potent immunogenicity.

\section{Conclusions}

In summary, this study suggested that SPI-1 T3SS could be exploited for cytosolic delivery of staphylococcal antigens SaEsxA and SaEsxB, to effectively activate antigenspecific humoral and cellular immune responses, and provide protection against $S$. aureus infection.

\section{Additional files}

Additional file 1: Table S1. Evaluation of humoral and mucosal immune responses against rSaEsXA and $r$ SaEsxB by ELISA. Readout at OD450 was shown. (XLSX $49 \mathrm{~kb}$ )

Additional file 2: Table S2. Evaluation of cellular immune response against rSaEsxA and rSaEsxB by ELISPOT. The spot-forming cells were counted using an immunospot reader system. The spot numbers were shown. (XLSX $41 \mathrm{~kb})$ 
Additional file 3: Table S3. Survival curves of vaccinated mice after lethal challenge with two clinical S. aureus strains. Mouse numbers were shown after challenge with S. aureus strains. (XLSX $35 \mathrm{~kb}$ )

\section{Abbreviations}

APCs: Antigen presenting cells; BHI: Brain Heart Infusion Broth; CAMRSA: Community-associated MRSA; CFU: Colony forming unit: ELISPOT: Enzyme-linked immunospot; ESAT-6: Early Secreted Antigenic Target-6; FBS: Fetal bovine serum; HRP: Horseradish peroxidase; IFNү: Interferon-gamma; LL-17A: Interleukine-17A; LB: Luria-Bertani; M. tuberculosis: Mycobacteria tuberculosis; MOI: Multiplicity of infection; MRSA: Methicillin-resistant S. aureus; MSSA: Methicillin-susceptible S. aureus; PEEC: Pathogen-elicited epithelial chemoattractant; PKC: Protein kinase C; PpagC: PagC promoter; S. aureus: Staphylococcus aureus; S. Typhimurium: Salmonella enterica subsp. enterica serotype Typhimurium; SFC: Spot-forming cells; slgA: Secretory IgA; SPI-1 T3SS: Salmonella Pathogenesis Island-1 Type 3 Secretion System; TCA: Trichloroacetic acid; TMB: Tetramethylbenzidine

\section{Funding}

This work was supported by the Research Fund for the Control of Infectious Diseases Commissioned Study of Food and Health Bureau of Hong Kong Government (RFCID, HK-09-01-21); Health and Medical Research Fund (HMRF, HKM-15-M09,14130742); a National Basic Research Program of China (973 Program, 2014CB745200) from the Ministry of Science and Technology of PRC, and by Shenzhen Peacock project (KQTD2015033117210153); the Shenzhen Science and Technology Innovation Committee Basic Science Research Grant (JCYJ20150629151046896)

\section{Availability of data and materials}

All data generated or analysed during this study are included in this published article and its Additional files.

\section{Authors' contributions}

$C X, B Z, Q L, B Y, J D H$ and $K Y Y$ designed the experiments. CX, BZ, JD and SA performed the experiments. CX analyzed the data. CX, QL and JDH drafted and revised the manuscript. KYY provided the key reagents and critical comments. All the authors read and approved the final manuscript.

\section{Ethics approval}

All animal experiments were approved by the Committee on the Use of Live Animals in Teaching and Research at the Laboratory Animal Unit of the University of Hong Kong (CULATR 3569-15). This study complies with relevant national and international guidelines.

\section{Competing interests}

The authors declare that they have no competing interests.

\section{Publisher's Note}

Springer Nature remains neutral with regard to jurisdictional claims in published maps and institutional affiliations.

\section{Author details}

'School of Biomedical Sciences, The University of Hong Kong, Hong Kong, SAR, China. ${ }^{2}$ Department of Microbiology, The University of Hong Kong, Hong Kong, SAR, China. ${ }^{3} \mathrm{HKU}$-Shenzhen Institute of Research and Innovation, Shenzhen, China. ${ }^{4}$ Shenzhen Institute of Advanced Technologies, Shenzhen, China.

\section{Received: 24 September 2017 Accepted: 19 April 2018}

Published online: 25 April 2018

\section{References}

1. Chen LM, et al. Optimization of the delivery of heterologous proteins by the Salmonella enterica serovar typhimurium type III secretion system for vaccine development. Infect Immun. 2006;74(10):5826-33.

2. Korea CG, et al. Staphylococcal Esx proteins modulate apoptosis and release of intracellular Staphylococcus aureus during infection in epithelial cells. Infect Immun. 2014;82(10):4144-53.
3. Kennedy AD, et al. Epidemic community-associated methicillin-resistant Staphylococcus aureus: recent clonal expansion and diversification. Proc Natl Acad Sci U S A. 2008;105(4):1327-32.

4. Abrahamian FM, Moran GJ. Methicillin-resistant Staphylococcus aureus infections. N Engl J Med. 2007;357(20):2090. author reply 2090

5. Gordon RJ, Lowy FD. Pathogenesis of methicillin-resistant Staphylococcus aureus infection. Clin Infect Dis. 2008;46(Suppl 5):S350-9.

6. Datta R, Huang SS. Risk of infection and death due to methicillin-resistant Staphylococcus aureus in long-term carriers. Clin Infect Dis. 2008;47(2):176-81.

7. Salgado-Pabon W, Schlievert PM. Models matter: the search for an effective Staphylococcus aureus vaccine. Nat Rev Microbiol. 2014;12(8):585-91.

8. Shinefield $\mathrm{H}$, et al. Use of a Staphylococcus aureus conjugate vaccine in patients receiving hemodialysis. N Engl J Med. 2002;346(7):491-6.

9. Fowler VG, et al. Effect of an investigational vaccine for preventing Staphylococcus aureus infections after cardiothoracic surgery: a randomized trial. JAMA. 2013;309(13):1368-78.

10. Weems, J.J., Jr., et al., Phase II, randomized, double-blind, multicenter study comparing the safety and pharmacokinetics of tefibazumab to placebo for treatment of Staphylococcus aureus bacteremia. Antimicrob Agents Chemother, 2006. 50(8): p. 2751-2755.

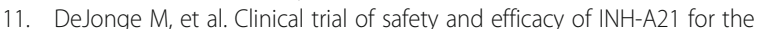
prevention of nosocomial staphylococcal bloodstream infection in premature infants. J Pediatr. 2007;151(3):260-5. 265 e1

12. Ohlsen $\mathrm{K}$, Lorenz U. Immunotherapeutic strategies to combat staphylococcal infections. Int J Med Microbiol. 2010;300(6):402-10.

13. Rupp ME, et al. Phase II, randomized, multicenter, double-blind, placebocontrolled trial of a polyclonal anti-Staphylococcus aureus capsular polysaccharide immune globulin in treatment of Staphylococcus aureus bacteremia. Antimicrob Agents Chemother. 2007;51(12):4249-54.

14. Spaulding AR, et al. Staphylococcal and streptococcal superantigen exotoxins. Clin Microbiol Rev. 2013;26(3):422-47.

15. Proctor RA. Challenges for a universal Staphylococcus aureus vaccine. Clin Infect Dis. 2012;54(8):1179-86.

16. Strandberg $\mathrm{KL}$, et al. Staphylococcal superantigens cause lethal pulmonary disease in rabbits. J Infect Dis. 2010;202(11):1690-7.

17. Spaulding AR, et al. Immunity to Staphylococcus aureus secreted proteins protects rabbits from serious illnesses. Vaccine. 2012;30(34):5099-109.

18. Spaulding AR, et al. Vaccination against Staphylococcus aureus pneumonia. J Infect Dis. 2014;209(12):1955-62.

19. Burts ML, et al. EsXA and EsxB are secreted by an ESAT-6-like system that is required for the pathogenesis of Staphylococcus aureus infections. Proc Natl Acad Sci U S A. 2005;102(4):1169-74.

20. Andersen $P$, et al. Recall of long-lived immunity to Mycobacterium tuberculosis infection in mice. J Immunol. 1995;154(7):3359-72.

21. Sorensen AL, et al. Purification and characterization of a low-molecular-mass T-cell antigen secreted by Mycobacterium tuberculosis. Infect Immun. 1995;63(5):1710-7.

22. Zhang BZ, et al. Recombinant ESAT-6-like proteins provoke protective immune responses against invasive Staphylococcus aureus disease in a murine model. Infect Immun. 2015;83(1):339-45.

23. Bagnoli F, et al. Vaccine composition formulated with a novel TLR7dependent adjuvant induces high and broad protection against Staphylococcus aureus. Proc Natl Acad Sci U S A. 2015;112(12):3680-5.

24. Monaci E, et al. MF59- and $\mathrm{Al}(\mathrm{OH}) 3$-Adjuvanted Staphylococcus aureus (4Cstaph) vaccines induce sustained protective humoral and cellular immune responses, with a critical role for effector CD4 T cells at low antibody titers. Front Immunol. 2015;6:439.

25. Mancini F, et al. One dose of Staphylococcus aureus 4C-staph vaccine formulated with a novel TLR7-dependent adjuvant rapidly protects mice through antibodies, effector CD4+ T cells, and IL-17A. PLoS One. 2016;11(1):e0147767.

26. Zheng SY, et al. Comparative immunological evaluation of recombinant Salmonella typhimurium strains expressing model antigens as live oral vaccines. BMC Immunol. 2012;13:54.

27. Russmann $\mathrm{H}$, et al. Delivery of epitopes by the Salmonella type III secretion system for vaccine development. Science. 1998;281(5376):565-8.

28. Konjufca $V$, et al. A recombinant attenuated Salmonella enterica serovar typhimurium vaccine encoding Eimeria acervulina antigen offers protection against E. Acervulina challenge. Infect Immun. 2006;74(12):6785-96.

29. Russmann $\mathrm{H}$, et al. Protection against murine listeriosis by oral vaccination with recombinant Salmonella expressing hybrid Yersinia type III proteins. J Immunol. 2001;167(1):357-65. 
30. Shams H, et al. Induction of specific CD8+ memory T cells and long lasting protection following immunization with Salmonella typhimurium expressing a lymphocytic choriomeningitis MHC class I-restricted epitope. Vaccine. 2001;20(3-4):577-85.

31. Evans DT, et al. Mucosal priming of simian immunodeficiency virus-specific cytotoxic T-lymphocyte responses in rhesus macaques by the Salmonella type III secretion antigen delivery system. J Virol. 2003;77(4):2400-9.

32. Singer $\mathrm{HM}$, et al. Selective purification of recombinant neuroactive peptides using the flagellar type III secretion system. MBio. 2012;3(3)

33. Darwin $\mathrm{KH}$, Miller $\mathrm{VL}$. Molecular basis of the interaction of Salmonella with the intestinal mucosa. Clin Microbiol Rev. 1999;12(3):405-28.

34. Georgiou G, Segatori L. Preparative expression of secreted proteins in bacteria: status report and future prospects. Curr Opin Biotechnol. 2005;16(5):538-45.

35. Wickner W, Schekman R. Protein translocation across biological membranes. Science. 2005;310(5753):1452-6.

36. Widmaier DM, et al. Engineering the Salmonella type III secretion system to export spider silk monomers. Mol Syst Biol. 2009;5:309.

37. Lee CA, et al. A secreted Salmonella protein induces a proinflammatory response in epithelial cells, which promotes neutrophil migration. Proc Natl Acad Sci U S A. 2000;97(22):12283-8.

38. Criss AK, et al. Regulation of Salmonella-induced neutrophil transmigration by epithelial ADP-ribosylation factor 6. J Biol Chem. 2001;276(51):48431-9.

39. Silva $\mathrm{M}$, et al. Salmonella typhimurium SipA-induced neutrophil transepithelial migration: involvement of a PKC-alpha-dependent signal transduction pathway. Am J Physiol Gastrointest Liver Physiol. 2004;286(6): G1024-31.

40. Zhou D, Mooseker MS, Galan JE. Role of the S. Typhimurium actin-binding protein SipA in bacterial internalization. Science. 1999;283(5410):2092-5.

41. Zhou D, Mooseker MS, Galan JE. An invasion-associated Salmonella protein modulates the actin-bundling activity of plastin. Proc Natl Acad Sci U S A. 1999:96(18):10176-81.

42. Hoiseth SK, Stocker BA. Aromatic-dependent Salmonella typhimurium are non-virulent and effective as live vaccines. Nature. 1981:291(5812):238-9.

43. Yu B, et al. A method to generate recombinant Salmonella typhi Ty21a strains expressing multiple heterologous genes using an improved recombineering strategy. Appl Microbiol Biotechnol. 2011;91(1):177-88.

44. Collazo CM, Galan JE. The invasion-associated type III system of Salmonella typhimurium directs the translocation of sip proteins into the host cell. Mol Microbiol. 1997:24(4):747-56.

45. Stone GW, et al. Multimeric soluble CD40 ligand and GITR ligand as adjuvants for human immunodeficiency virus DNA vaccines. J Virol. 2006:80(4):1762-72.

46. Cardenal-Munoz E, Ramos-Morales F. Analysis of the expression, secretion and translocation of the Salmonella enterica type III secretion system effector SteA. PLoS One. 2011;6(10):e26930.

47. Dunstan SJ, Simmons CP, Strugnell RA. Use of in vivo-regulated promoters to deliver antigens from attenuated Salmonella enterica var. Typhimurium. Infect Immun. 1999:67(10):5133-41.

48. Kubori T, Galan JE. Salmonella type III secretion-associated protein InvE controls translocation of effector proteins into host cells. J Bacteriol. 2002;184(17):4699-708.

49. Corthesy B. Multi-faceted functions of secretory lgA at mucosal surfaces. Front Immunol. 2013;4:185.

50. Lin $L$, et al. Th1-Th17 cells mediate protective adaptive immunity against Staphylococcus aureus and Candida albicans infection in mice. PLoS Pathog. 2009:5(12):e1000703.

51. Misstear $\mathrm{K}$, et al. Targeted nasal vaccination provides antibody-independent protection against Staphylococcus aureus. J Infect Dis. 2014;209(9):1479-84.

52. van Kessel KP, Bestebroer J, van Strijp JA. Neutrophil-mediated phagocytosis of Staphylococcus aureus. Front Immunol. 2014;5:467.

53. Pathangey $L$, et al. Effect of expression level on immune responses to recombinant oral Salmonella enterica serovar typhimurium vaccines. Vaccine. 2009:27(20):2707-11.

54. Juarez-Rodriguez MD, et al. Live attenuated Salmonella vaccines displaying regulated delayed lysis and delayed antigen synthesis to confer protection against Mycobacterium tuberculosis. Infect Immun. 2012;80(2):815-31.

55. Cooper AM, et al. Disseminated tuberculosis in interferon gamma genedisrupted mice. J Exp Med. 1993;178(6):2243-7.

56. Flynn $J \mathrm{~L}$, et al. An essential role for interferon gamma in resistance to Mycobacterium tuberculosis infection. J Exp Med. 1993;178(6):2249-54.
57. Lowy FD. Is Staphylococcus aureus an intracellular pathogen? Trends Microbiol. 2000:8(8):341-3.

58. Fraunholz M, Sinha B. Intracellular Staphylococcus aureus: live-in and let die. Front Cell Infect Microbiol. 2012;2:43.

59. Kubica $M$, et al. A potential new pathway for Staphylococcus aureus dissemination: the silent survival of S. Aureus phagocytosed by human monocyte-derived macrophages. PLoS One. 2008;3(1):e1409.

60. Paul WE. Cell-mediated cytotoxicity. Fundamental immunology. 7th ed; 2012

61. Broker BM, Mrochen D, Peton V. The T cell response to Staphylococcus aureus. Pathogens. 2016;5(1)

62. Chen K, et al. Th17 cells mediate clade-specific, serotype-independent mucosal immunity. Immunity. 2011;35(6):997-1009.

63. Hsu HC, et al. Interleukin 17-producing T helper cells and interleukin 17 orchestrate autoreactive germinal center development in autoimmune BXD2 mice. Nat Immunol. 2008:9(2):166-75.

64. Alcorn JF, Crowe CR, Kolls JK. TH17 cells in asthma and COPD. Annu Rev Physiol. 2010;72:495-516.

65. Islander $U$, et al. Superantigenic Staphylococcus aureus stimulates production of interleukin-17 from memory but not naive T cells. Infect Immun. 2010:78(1):381-6.

\section{Ready to submit your research? Choose BMC and benefit from:}

- fast, convenient online submission

- thorough peer review by experienced researchers in your field

- rapid publication on acceptance

- support for research data, including large and complex data types

- gold Open Access which fosters wider collaboration and increased citations

- maximum visibility for your research: over $100 \mathrm{M}$ website views per year

At BMC, research is always in progress.

Learn more biomedcentral.com/submissions 